\title{
Salivary gland function in thyroid cancer patients with radioiodine administration history
}

\author{
Eva Krcalovaa ${ }^{\mathrm{a}, \mathrm{b}}$, Jiri Horacek ${ }^{\mathrm{b}, c}$, Filip Gabalec ${ }^{\mathrm{b}, c}$, Pavel Zak ${ }^{\mathrm{b}, c}$, Jiri Dolezal ${ }^{\mathrm{a}}$
}

\begin{abstract}
Aim. Radioiodine (RAI) improves survival in patients with locally advanced or metastatic differentiated thyroid carcinoma (DTC). Although there has been an ongoing debate on RAl-induced salivary gland damage, published data have been inconsistent. Therefore, the purpose of our study was to compare salivary gland function in intermediate and high risk DTC patients after single or repeated RAI treatment with their age- and sex-matched RAI-naive counterparts. Methods. Uptake and excretion of parotid and submandibular glands were quantitatively evaluated using ${ }^{99 \mathrm{~m} T c-p e r-}$ technetate salivary gland scintigraphy in 23 patients previously treated with RAl. Patients (median $9.25 \mathrm{GBq}^{131}$ I-Nal; Q1-Q3: 5.55-16.65; range: 5.55-27.5) were divided into subgroups according to previously administered ${ }^{131}$ I-Nal activity using cut-off values $5.55 \mathrm{GBq}$ and $9.25 \mathrm{GBq}$. Their salivary gland scintigraphy results were compared with RAl-naive patients using Mann-Whitney test.

Results. Compared to RAl-naive patients, parotid glands pertechnetate uptake was significantly lower in those treated with $>9.25 \mathrm{GBq}(P=0.034)$ and parotid glands excretion fraction was already decreased with RAl activities $>5.55 \mathrm{GBq}$ $(P=0.031)$. In submandibular glands, no statistically significant difference in either function was observed even with RAl activity > 9.25 GBq.

Conclusion. Our data suggest that RAl therapy using activities $\leq 5.55 \mathrm{GBq}$ does not substantially decrease saliva production. Activities $>5.55 \mathrm{GBq}$ may lead to significant decrease in parotid excretion, and activities $>9.25 \mathrm{GBq}$ also diminish parotid uptake. Surprisingly, submandibular glands, providing majority of seromucinous saliva under basal condition, seem to be unaffected even by RAl activities above $9.25 \mathrm{GBq}$.
\end{abstract}

Key words: radioiodine, thyroid cancer, salivary gland dynamic scintigraphy, ${ }^{99 m}$ Tc-pertechnetate

Received: March 8, 2019; Revised: May 14, 2019; Accepted: May 20, 2019; Available online: June 17, 2019

https://doi.org/10.5507/bp.2019.023

(c) 2020 The Authors; https://creativecommons.org/licenses/by/4.0/

${ }^{a}$ Department of Nuclear Medicine, University Hospital Hradec Kralove, Sokolska 581, 50005 Hradec Kralove, Czech Republic

${ }^{b}$ Academic Department of Internal Medicine, Charles University, Faculty of Medicine in Hradec Kralove, Sokolska 581,50005 Hradec Kralove, Czech Republic

${ }^{c} 4^{\text {th }}$ Department of Internal Medicine, Charles University, Faculty of Medicine in Hradec Kralove and University Hospital Hradec Kralove, Sokolska 581, 50005 Hradec Kralove, Czech Republic

Corresponding author: Eva Krcalova, e-mail: krcalova.e@gmail.com

\section{INTRODUCTION}

Radioactive iodine 131 (RAI) has been an important part of differentiated thyroid cancer (DTC) treatment. As a combined beta and gamma emitter, RAI enables not only radioiodine remnant ablation (RRA) following total thyroidectomy (i.e. molecular radiotherapy) but also provides a precise, image-based staging of the disease thanks to post-ablation RAI whole body scan ${ }^{1}$. Whereas in low risk disease there is a trend towards using lower activities or even RRA omission, in patients with locally advanced or metastatic DTC an adequately high (and safely achievable) activity for RRA or RAI therapy is being sought. Successful RRA in high risk patients guarantees them equally long recurrence-free survival as in successfully ablated low risk ones ${ }^{2-4}$.

Although the DTC therapy using RAI has undoubtedly been a success story of nuclear medicine, significantly improving survival rates mainly in advanced disease ${ }^{1,5,6}$, there has been an ongoing debate on RAI minor side effects, namely RAI-induced salivary gland damage ${ }^{7-10}$.
Salivary glands are susceptible to RAI-induced damage due to sodium/iodide symporter (NIS) expressed on ductal cells surface. RAI is, therefore, trapped in these cells in the same way as in thyroid cells ${ }^{11}$. As saliva enables taste sensation and plays important role in oral microflora balance maintenance, in enamel remineralization, proper food digestion as well as in a wide variety of defensive processes and oral mucosa protection ${ }^{12}$, xerostomia may importantly affect the quality of life.

Some sequelae of RAI-induced xerostomia (such as problems with chewing and swallowing and an increase in caries rate) have been reported in retrospective studies based on interviews, questionnaires and hospital records describing subjective symptoms ${ }^{13-16}$. However, methodology of some of these frequently cited papers makes their conclusions rather questionable ${ }^{10}$. Especially the long intervals (ranging from 1 up to 21 years) between RAI administration and patients interviewing in the study by Alexander et al. ${ }^{14}$ may be a matter of concern. Additionally, Walter et al. ${ }^{13}$ analysed long-term impact of RAI administration on oral health. The authors identified 
RAI administration as an independent predictor of sialadenitis and xerostomia. However, the independent risk factor for new caries after RAI was not only xerostomia, but also prior caries - indicating important influence of patient's oral hygiene. Surprisingly, only administered RAI activity was found to be an independent predictor of higher tooth extraction rate - this is confusing, as neither salivary gland uptake of RAI, nor sialadenitis, nor xerostomia were identified as independent predictors of tooth extractions. Considering this, a relation between RAI activity and tooth extraction remains at best elusive ${ }^{10}$.

An objective assessment of the salivary gland RAIinduced injury may be enabled by dosimetry. Recent dosimetric studies using either ${ }^{131} \mathrm{I}-\mathrm{NaI}$ planar scans and salivary gland CT (used for salivary gland volume determination) or ${ }^{124} \mathrm{I}-\mathrm{NaI} \mathrm{PET} / \mathrm{CT}$ proved that average salivary gland dose per commonly administered RAI ablation activity is about one order of magnitude lower than the external beam radiotherapy dose necessary to induce salivary gland radiation injury ${ }^{17,18}$. Nevertheless, continuously decreasing RAI uptake in salivary glands (marker of parenchymal integrity) during the repeated RAI administrations has been observed ${ }^{18}$.

In recent guidelines, however, there is no absorbeddose constraint for salivary glands ${ }^{19}$ reported. Moreover, the 2015 American Thyroid Association guidelines, commonly used by endocrinologists, lacks recommendation regarding salivary gland function monitoring or protection $^{20}$.

Dynamic salivary gland scintigraphy (SGS) with ${ }^{99 \mathrm{~m}} \mathrm{Tc}-$ pertechnetate is another option for objective salivary gland function evaluation ${ }^{21}$. This non-invasive method allows quantifying both the glandular uptake (reflecting parenchymal integrity) and the excretion fraction (reflecting ability to release an adequate saliva volume after a gustatory stimulus) for each gland. Thus far published studies ${ }^{22-26}$ using SGS were, however, somewhat contradictory in the observed frequency, extent and severity of RAIinduced salivary gland injury as well as in the relationship between the injury and cumulative RAI activity.

Therefore, the purpose of this study was to assess objectively, using SGS and precise quantification, possible changes in salivary gland function in patients with a history of RAI treatment in comparison with their RAI-naive age- and sex-matched counterparts. We focused on intermediate or high risk patients treated with activities up to 9.25 $\mathrm{GBq}$ and on those with advanced disease treated with cumulative activities above $9.25 \mathrm{GBq}$.

\section{PATIENTS AND METHODS}

\section{Patients}

We performed SGS in 23 patients previously treated with RAI activities ranging from 5.55 to $27.75 \mathrm{GBq}$ ${ }^{131} \mathrm{I}-\mathrm{NaI}$ and compared their salivary glands performance with their RAI-naive age- and sex-matched counterparts (unpaired design). No statistically significant difference in age between the groups was observed ( $P=0.468$, MannWhitney test) and sex ratios were exactly equal. Details are summarized in Table 1. Both RAI-naive and previously treated patients were hypothyroid at the time of SGS performed the day before their (first or repeated) RAI treatment. Patients did not receive sialogogues dur-

Table 1. Characteristics of patients with radioiodine administration history and their RAI-naive couterparts.

\begin{tabular}{|c|c|c|c|c|}
\hline \multirow{2}{*}{$\begin{array}{l}\text { Parameter } \\
\text { Gender, } \mathrm{n}\end{array}$} & \multicolumn{2}{|c|}{$\begin{array}{l}\text { Previously treated patients } \\
\qquad(\mathrm{n}=23)\end{array}$} & \multicolumn{2}{|c|}{$\begin{array}{l}\text { RAI-naive patients } \\
\qquad(\mathrm{n}=23)\end{array}$} \\
\hline & & & & \\
\hline male & 7 & & 7 & \\
\hline female & 16 & & 16 & \\
\hline \multicolumn{5}{|l|}{ Age (years) } \\
\hline median (Q1-Q3) & 60 & $(44-76)$ & 60 & $(44-70)$ \\
\hline \multicolumn{5}{|l|}{ Histology, n } \\
\hline PTC & 19 & & $20+2 *$ & \\
\hline FTC & 1 & & 3 & \\
\hline Oncocytic & 3 & & 0 & \\
\hline \multicolumn{5}{|l|}{ Stage (AJCC 2009), n } \\
\hline Stage I & 8 & & 13 & \\
\hline Stage II & 1 & & 1 & \\
\hline Stage III & 3 & & 7 & \\
\hline Stage IVa & 8 & & 2 & \\
\hline Stage IVb & 0 & & 0 & \\
\hline Stage IVc & 3 & & 0 & \\
\hline \multicolumn{5}{|l|}{$\begin{array}{l}\text { Cumulative RAI activity } \\
\text { (GBq) }\end{array}$} \\
\hline median (Q1-Q3) & 9.25 & $(5.55-16.65)$ & - & - \\
\hline
\end{tabular}

FTC - follicular thyroid cancer, PTC - papillary thyroid cancer, AJCC - American Joint Committee on Cancer, RAI - Radioactive iodine ${ }^{131}$ I-NaI, * DTC tumour duplicity 

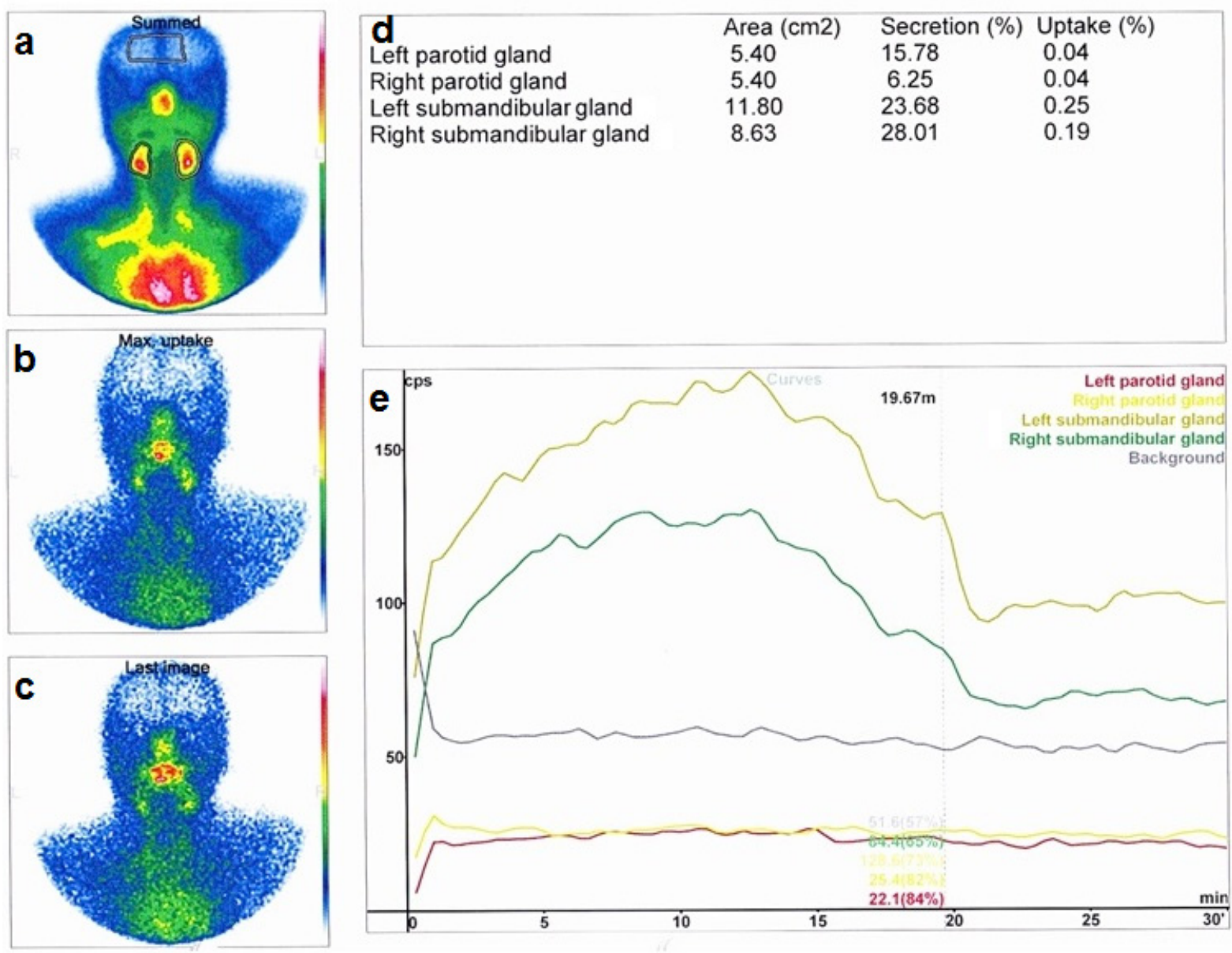

Fig. 1. Physiological uptake and excretion of ${ }^{99 \mathrm{~m}}$ Tc-pertechnetate in submandibular salivary glands and parotid glands dysfunction detected by dynamic salivary gland scintigraphy. Summed image (a) obtained from all frames depicts salivary glands (parotid glands are only faintly seen), submandibular glands ROIs and ROI for background correction placed over the brain. Picture (b) shows maximal radiotracer uptake in the salivary glands and (c) shows radiotracer activity in salivary glands 10 minutes after salivary glands gustatory stimulation. Values in (d) represent calculated uptake and excretion fractions and (e) shows time-activity curves for each salivary gland.

ing RAI therapy. None of the patients had a history of salivary gland disease or head and neck external beam radiotherapy. The group of 23 patient pairs was subsequently divided into subgroups according to the cumulative activity administered to the previously treated patients. Salivary gland uptake and excretion fractions changes were assessed using two cut-off values: $5.55 \mathrm{GBq}$ (5.55 GBq: 10 patient pairs; > 5.55 GBq: 13 patient pairs) and $9.25 \mathrm{GBq}$, once sorting those treated with cumulative activity of $9.25 \mathrm{GBq}$ into higher activity group (< 9.25GBq: 11 patient pairs; $\geq 9.25 \mathrm{GBq}: 12$ patient pairs) once into the lower one $(\leq 9.25 \mathrm{GBq}$ : 14 patient pairs; $>$ 9.25 GBq: 9 patient pairs).

\section{Dynamic salivary gland scintigraphy}

The salivary gland scintigraphy validated protocol described by Klutmann et al. ${ }^{21}$ was used for salivary gland functional parameters assessment. Imaging was performed using a single headed gammacamera $\mathrm{MB}$ 9200 (Gamma Müvek, Budapest, Hungary) equipped with a parallel hole, low energy high resolution collimator. Dynamic scan acquisition (matrix 128x128x16, 90 frames, each per 20 seconds, photopeak centered at 140
$\mathrm{keV}$ with $10 \%$ symmetric window) started simultaneously with $185 \mathrm{MBq}{ }^{99 \mathrm{~m}}$ Tc-pertechnetate intravenous administration. Patients laid supine with head stabilised using a U-shaped support during the data acquisition. Lemon juice ( $3 \mathrm{~mL}$, Realemon, Meroso Foods N.V., Ramsdonk, Belgium), used as a gustatory stimulus, was administered per os 20 minutes after radiotracer injection. Regions of interest (ROI) were drawn over each parotid and submandibular gland and one for background correction was created over the brain. The time-activity curves of each ROI were subsequently generated (Fig. 1).

Salivary gland uptake was calculated as the background subtracted percentage of total ${ }^{99 \mathrm{~m}} \mathrm{Tc}$-pertechnetate administered activity measured at the time of peak activity. Salivary gland excretion fraction was calculated as the difference between pre-stimulation and post-stimulation uptake expressed as the percentage of pre-stimulation one $^{21}$.

In each patient, the average uptake and excretion fraction for left and right parotid gland and for left and right submandibular gland were calculated, and the average values for each gland were further evaluated. 
Table 2. Comparison of ${ }^{99 \mathrm{~m}} \mathrm{Tc}$-pertechnetate uptake and secretion in salivary glands between patients with RAI administration history (all activities, $n=23$ ) versus their age- and sex-matched RAI-naive counterparts $(n=23)$.

\begin{tabular}{lccc}
\hline Variable & RAI-naive & RAI administration history & $P$ \\
\hline Parotid gland & & & $0.13(0.07-0.19)$ \\
$\quad$ Uptake (\%) & $0.16(0.10-0.19)$ & $35.5(22.6-50.8)$ & 0.051 \\
$\quad$ Excretion fraction (\%) & $46.0(37.2-56.9)$ & & 0.956 \\
Submandibular gland & & & 1.000 \\
$\quad$ Uptake (\%) & $0.15(0.13-0.20)$ & $32.5(24.1-42.5)$ & \\
$\quad$ Excretion fraction (\%) & $34.3(22.5-41.1)$ & & \\
\hline
\end{tabular}

Values are median (Q1-Q3), $P$ values from Mann-Whitney test
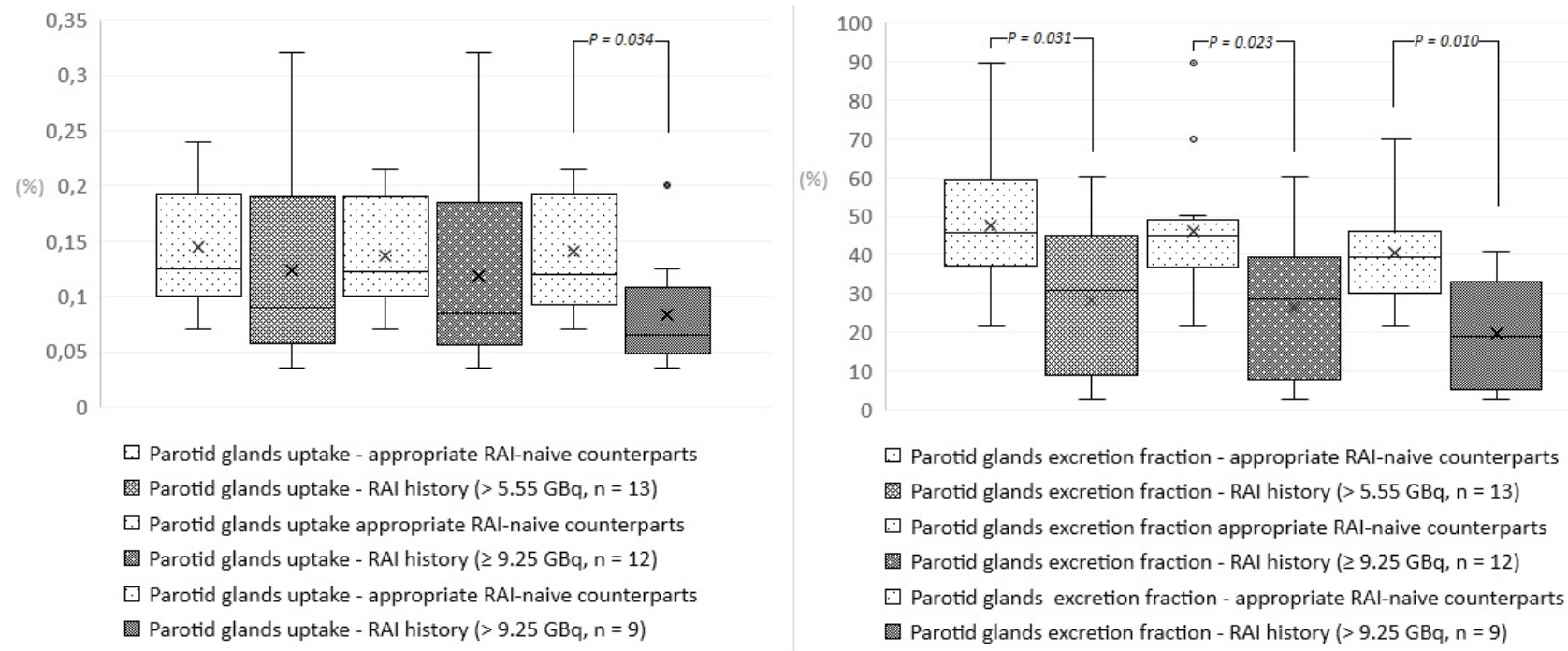

Fig. 2. Parotid glands uptake (left) and excretion fraction (right) in patients treated with RAI activities $>5.55 \mathrm{GBq}, \geq 9.25 \mathrm{GBq}$ and $>9.25 \mathrm{GBq}$. $P$ values from Mann-Whitney test.

\section{Statistical analysis}

For statistical analysis, SigmaStat software package (Jandel Corporation, San Rafael, CA, USA), version 3.1, was used. The measured values are presented as medians and $1^{\text {st }}$ and $3^{\text {rd }}$ quartile (Q1-Q3) in parentheses, due to non-normal data distribution. The comparisons of pooled parotid and pooled submandibular glands uptake and excretion fraction in previously treated versus RAI-naive patients were performed using non-parametric Mann-Whitney test. A $P$ value of $<0.05$ was considered to be statistically significant. Our study was approved by University Hospital Hradec Králové Ethical Committee and written informed consent was obtained from all patients.

\section{RESULTS}

In 23 patients ( 7 males, 16 females, median age 60 years; Q1-Q3: 44-76) with previous RAI administration history, SGS was performed at least 4 months (median 11.9 month; Q1-Q3: 7.8-25.6) after their last RAI treatment (administered activity median $9.25 \mathrm{GBq}{ }^{131} \mathrm{I}-\mathrm{NaI}$; Q1-Q3: 5.55-16.65, range 5.55-27.5). RAI-naive counterparts of the same gender and similar age (median 60 years; Q1-Q3: 44-70) were matched to them.
Compared to their RAI-naive counterparts, the whole group of previously treated patients was not significantly different in either gland uptake (reflecting parenchymal integrity) or excretion fraction (reflecting the adequate secretory response to gustatory stimulus). The results are presented in Table 2. While the uptake to submandibular glands was somewhat higher in those with RAI administration history, the uptake to parotid glands was somewhat lower. The excretion fractions in both glands were nonsignificantly lower in previously treated patients, and for parotid glands the difference was close to the significance level $(P=0.051)$.

Therefore, we attempted to assess a possible dependence of this borderline effect on the administered activity. We divided the group according to the activities 5.55 GBq ${ }^{131} \mathrm{I} \mathrm{Na-I}$ and $9.25 \mathrm{GBq}^{131} \mathrm{I} \mathrm{Na}-\mathrm{I}$ (once included into the lower, once into the higher activity group).

In all higher activity subgroups (activities $>5.55 \mathrm{GBq}$, $\geq 9.25 \mathrm{GBq}$ and $>9.25 \mathrm{GBq}$ ) a statistically significant decrease in parotid gland excretion fraction was detected ( $P=0.031, P=0.023$ and 0.01 , respectively). Nevertheless, statistically significant decrease in parotid glands uptake was detected only in the subgroup treated with the highest cumulative activity $(P=0.034)$. Results are summarised in Fig. 2. On the other hand, submandibular glands uptake 


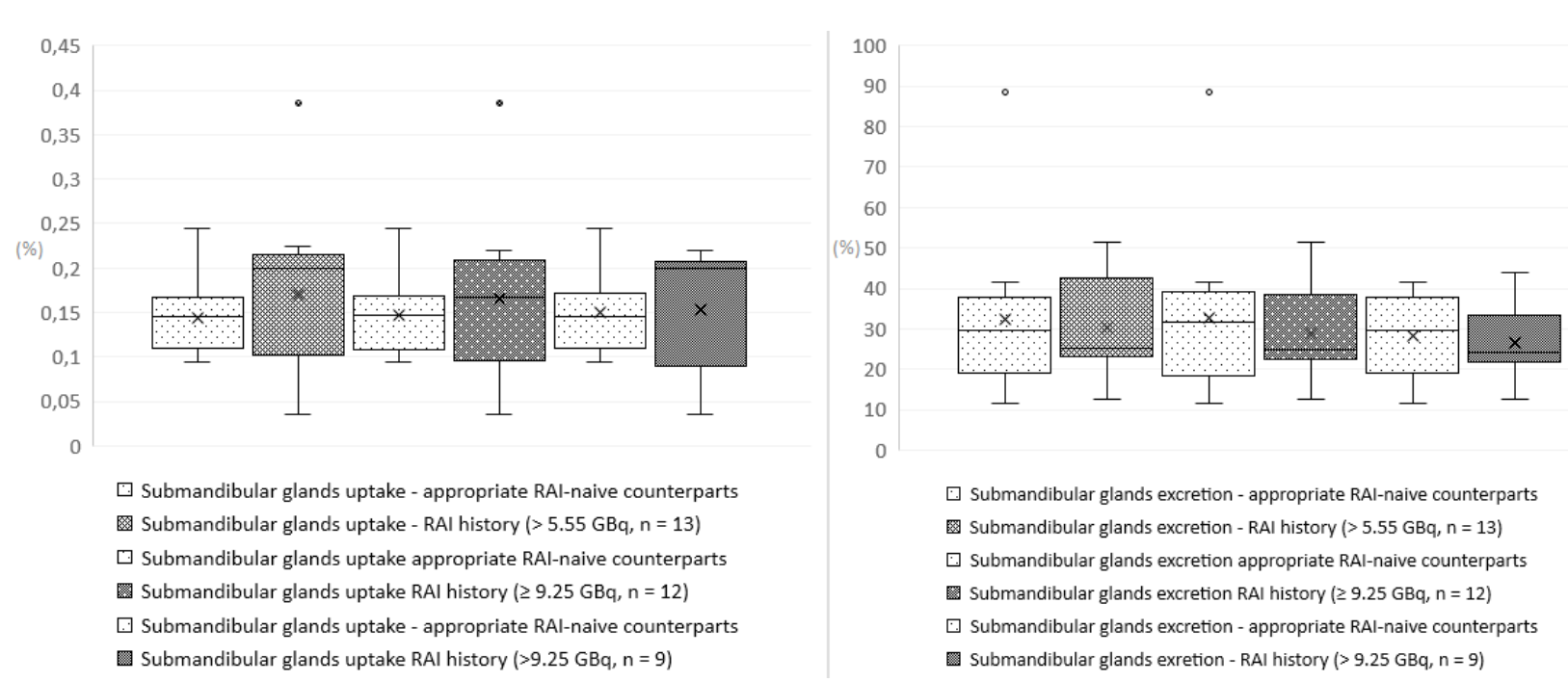

Fig. 3. Submandibular glands uptake (left) and excretion fraction (right) in patients treated with RAI activities $>5.55 \mathrm{GBq}, \geq 9.25$ $\mathrm{GBq}$ and $>9.25 \mathrm{GBq}$ were similar.

and excretion fractions were similar to RAI-naive patients (Fig. 3).

\section{DISCUSSION}

Our data suggest that repeated RAI administration (with cumulative activities $>9.25 \mathrm{GBq}$, ranging from 11.10 to $27.75 \mathrm{GBq}$ ) induce significant parotid gland impairment in terms of both parenchymal dysfunction and decreased saliva excretion. However, the decrease in parotid glands excretion fraction was observed with lower activities already. Indeed, excluding 10 patients previously treated with the lowest activity $(5.55 \mathrm{GBq})$ from the whole group, i.e. in 13 patients with higher activities, the decrease in excretion fraction became significant. These findings suggest that RAI activity cut-off value for RAIinduced parotid glands excretion decrease may be even lower than 9.25 GBq, but above 5.55 GBq. Nevertheless, due to small number of patients we are unable to perform more detailed analysis and set more precise cut-off value. Interestingly, submandibular salivary gland uptake and excretory function remained intact in both subgroups previously treated with lower or higher activity.

One of the first attempts using SGS for RAI-induced salivary gland damage quantification was performed by Bohuslavizki et al. in 1996 (ref. ${ }^{22}$ ). The authors compared salivary gland uptake in 106 patients before and 3 months after RAI administration (for both benign diseases and thyroid cancer) with age and sex matched counterparts and observed severe and statistically significant decrease in parotid as well as in submandibular glands uptake in 6 patients previously treated with cumulative activities of $24 \mathrm{GBq}{ }^{131} \mathrm{I}-\mathrm{NaI}$. Mild (but also statistically significant) changes in salivary glands uptake were, however, observed in all patients. Surprisingly, 15 patients previously treated with activities ranging from 1.4-1.85 GBq had similar extent of salivary gland uptake decrease to those previously treated with 6-9 GBq $(n=5)$ and both subgroups were affected more than 12 patients previously treated with
$3 \mathrm{GBq}$. This is in contradiction not only with our results, but also with Wu et al. and partially with Maruoka et al. papers reporting on 194 and 279 patients, respectively ${ }^{25,26}$.

None of these studies proved submandibular gland uptake decrease in patients treated with activities up to 7.6 GBq $\left(\right.$ ref. $^{26}$ ), resp.22.2 GBq (ref. $\left.{ }^{25}\right)$. However, statistically significant decline in parotid glands uptake was observed in patients treated with activities above $11.1 \mathrm{GBq}$ in $\mathrm{Wu}$ et al. study ${ }^{25}$ and in all patients in Maruoka et al. study ${ }^{26}$.

The role of administered activity regarding to RAI induced salivary gland injury remained unclear also in the study by Almeida et al. ${ }^{23}$. They quantitatively analysed SGS results in 182 patients; 76 of them RAI-naive and 106 after RAI treatment using activities ranging from 1.11 to 16.65 GBq ${ }^{131} \mathrm{I}$-NaI. They evaluated uptake and excretion both in parotid and submandibular glands and disclosed a strong association of RAI treatment with decreased parotid excretion using univariate analysis and multiple linear regression. Subsequently, the authors divided patients into two subgroups according to administered activity of 5.55 GBq for activity-dependence analysis. Rather surprisingly and discordantly to recent studies ${ }^{25,26}$, no such dependence was observed. Unfortunately, the activities used in the higher activities subgroup are not explicitly described in the paper. Contrary to parotid glands excretion, parotid gland uptake and both submandibular gland uptake and excretion were not affected by RAI treatment, which corresponds to our data.

Semi-quantitative analysis of SGS represents another option in RAI-induced salivary gland injury assessment. Solans et al. ${ }^{24}$ were among the first who used semi-quantitative scoring. The authors prospectively evaluated 79 patients for 3 years after one or more RAI administrations and observed incredibly high incidence of salivary gland impairment assessed by SGS (40 cases during the first year of follow-up, and 3 and 28 in the second and third year, respectively). However, their methodology had several flaws. First, the evaluated group was considerably heterogeneous, including patients with Graves-Basedow's disease (i.e. without total thyroidectomy). The adminis- 
tered activities then unsurprisingly ranged from 0.9 to $18.5 \mathrm{GBq}{ }^{131} \mathrm{I}-\mathrm{NaI}$, and no attempt to analyse the salivary gland impairment regarding to administered activity was made. Secondly, the semi-quantitative scoring used in their study did not reflect the changes in parotid and submandibular glands separately although this seems to be of great clinical importance. And finally, the excretion fraction assessment method was not specified at all. Salivary gland excretion fraction assessment using long (60 second) frames and no gustatory stimulus seems to be rather unreliable. All in all, results of the aforementioned study are contradictory to recently published papers.

$\mathrm{Wu}$ et al. ${ }^{25}$ analysed salivary gland function in a total of 368 patients with DTC who underwent total thyroidectomy. Of them, 174 did not received RAI after the operation (control group), 78 patients received activities $\leq 5.5$ $\mathrm{GBq}{ }^{131} \mathrm{I}-\mathrm{NaI}, 35$ patients were treated with activities $>5.5$ and $\leq 11.1 \mathrm{GBq}^{131} \mathrm{I}-\mathrm{NaI}, 48$ patients received cumulative activities $>11.1$ and $\leq 22.2 \mathrm{GBq}^{131} \mathrm{I}$-NaI and 33 patients were treated with cumulative activities higher than 22.2 $\mathrm{GBq}{ }^{131} \mathrm{I}-\mathrm{NaI}$.

Consistently with our results, they proved that salivary glands impairment depends on cumulative administered activity and that excretory function of salivary glands seems to be the most sensitive parameter of RAI-induced injury. They did not observe significant difference in parotid as well as in submandibular glands in either function in subgroup treated with activities up to $5.5 \mathrm{GBq}$, similarly, no such statistically significant changes were observed in our subgroup treated with activity of 5.5 GBq. Moreover, the decline in parotid glands uptake was statistically significant only in patients treated with cumulative RAI activities $\geq 11.1 \mathrm{GBq}$. Similarly resistant parotid glands uptake was observed in our study. However, statistically significant decrease in parotid glands excretion fraction was reported in the subgroup treated with activities $>5.55$ and $\leq 11.1 \mathrm{GBq}$. Likewise, our data suggest that RAI activity cut-off for inducing parotid gland excretion fraction decrease is above $5.55 \mathrm{GBq}$. Nevertheless, submandibular salivary glands uptake and even secretion seemed to be more resistant than parotid gland function in Wu et al. study, which is in accordance with our data.

The most recent study assessing salivary gland changes after RAI treatment using SGS was published in 2017 by Maruoka et al. ${ }^{26}$. Authors analysed changes in salivary glands function in 279 patients who underwent SGS before and $12 \pm 1$ month after last RAI administration. They simultaneously evaluated presence of subjective symptoms of dryness in the mouth. Salivary glands uptake and excretion evaluation was based on 3-point-scale scoring system. Intensity of tracer uptake was compared with background activity ( 2 points for normal intensity uptake, 1 point for decreased uptake and 0 point when uptake in gland was similar to background). The excretion fraction was measured in the same manner as in our study, and difference between pre- and post-treatment results was subsequently calculated. Decrease less than $10 \%$ was assigned 2 points, decrease between 11 and 99\% was for 1 point and complete loss of excretory function was for 0 points. This score was calculated for each glandular type and each function.

In patients treated with higher cumulative activities ( $\mathrm{n}=86$, median of administered activity $13.6 \mathrm{GBq}{ }^{131} \mathrm{I}-\mathrm{NaI}$; range: 10.0-33.9) and those with post-treatment dry mouth symptoms $(n=43$, median of administered activity 12.1 GBq ${ }^{131} \mathrm{I}-\mathrm{NaI}$; range: 7.6-33.7), both parotid and submandibular uptake and excretion scores were significantly lower than in the pre-treatment group. Nevertheless, in patients treated with lower cumulative activities $(n=193$, median of administered activity $4.0 \mathrm{GBq}{ }^{131} \mathrm{I}-\mathrm{NaI}$; range: 3.7-9.5) and asymptomatic ones $(n=236$, median of administered activity $7.4 \mathrm{GBq}{ }^{131} \mathrm{I}-\mathrm{NaI}$; range: $3.7-33.9$ ), submandibular gland uptake and excretion scores were preserved.

This corroborates our observation that submandibular glands are rather resistant to RAI-induced injury. Considering that submandibular salivary glands provide more than two thirds of daily saliva production ${ }^{27}$, this finding is of great clinical importance. Moreover, submandibular glands produce mixed, seromucinous saliva, containing both viscous and liquid components ${ }^{12}$. In addition, predictive analysis for dry mouth symptoms revealed decreased submandibular functional score (i.e. sum of submandibular uptake and excretion score) as the factor with highest predictive value for dry mouth symptoms ${ }^{26}$, which emphasises the crucial importance of submandibular glands function.

However, the decrease both in submandibular uptake and excretion score in patients treated with high RAI activities (median of administered activity $13.6 \mathrm{GBq}{ }^{131} \mathrm{I}-\mathrm{NaI}$; range: 10.0-33.9) observed by Maruoka et al. ${ }^{26}$ is in contrast with our and $\mathrm{Wu}$ et al. results ${ }^{25}$. This discrepancy seems to be influenced by rather strict semi-quantitative scoring system for salivary gland excretion function (salivary gland secretion score was already lowered with $11 \%$ salivary gland secretion decrease) and the semi-quantitative nature of the study ${ }^{26}$. The latter seems to be true also for lower parotid gland uptake score observed in this study in patients treated with activities ranging from 3.79.5 GBq $\left(\right.$ ref. $\left.^{26}\right)$.

Statistically significant decrease in parotid excretion scores in patients treated with activities up to $9.5 \mathrm{GBq}$ in Maruoka et al. study ${ }^{26}$ is consistent with our observation that RAI activity inducing statistically significant decrease in parotid gland excretion fraction may be probably lower than $9.25 \mathrm{GBq}$.

\section{CONCLUSION}

Our findings suggest that RAI administration using activities up to $5.55 \mathrm{GBq}$ does not substantially decrease saliva production. On the other hand, higher activities may lead to a significant decrease in parotid excretion, and activities $>9.25 \mathrm{GBq}$ also diminish parotid uptake. Surprisingly, submandibular glands, producing majority of seromucinous saliva (under basal conditions), seem to be unaffected even in patients treated with RAI activities 
higher than $9.25 \mathrm{GBq}$, which is important for their oral health maintenance and the quality of life.

Acknowledgement: This work was supported by the programme PROGRES Q40-14.

Author contributions: EK: performed and evaluated all salivary gland scintigraphies and wrote the manuscript; JH: performed statistical analysis, contributed substantially to the manuscript and revised it critically for important intellectual content; FG, PZ, JD: gave important clinical advice and revised the manuscript.

Conflict of interest statement: None declared.

\section{REFERENCES}

1. McCready VR. Radioiodine - the success story of Nuclear Medicine. Eur J Nucl Med Mol Imaging 2017;44(2):179-2.

2. Verburg FA, Stokkel MPM, Düren C, Verkooijen RBT, Mäder U, van Isselt JW, Marlowe RJ, Smit JW, Reiners C, Luster M. No survival difference after successful 131 l ablation between patients with initially low-risk and high-risk differentiated thyroid cancer. Eur J Nucl Med Mol Imaging 2010;37(2):276-3.

3. Verburg FA, Luster M, Giovanella L, Lassmann M, Chiesa C, Chouin N, Flux G. The "reset button" revisited: why high activity 131 I therapy of advanced differentiated thyroid cancer after dosimetry is advantageous for patients Eur J Nucl Med Mol Imaging 2017;44(6):915-7.

4. Lassmann M, Hänscheid H, Chiesa C, Hindorf C, Flux G, Luster M; EANM Dosimetry Committee. EANM Dosimetry Committee series on standard operational procedures for pre-therapeutic dosimetry I: blood and bone marrow dosimetry in differentiated thyroid cancer therapy. Eur J Nucl Med Mol Imaging 2008;35(7):1405-2.

5. Durante C, Haddy N, Baudin E, Leboulleux S, Hartl D, Travagli JP, Caillou B, Ricard M, Lumbroso JD, De Vathaire F, Schlumberger M. Long-term outcome of 444 patients with distant metastases from papillary and follicular thyroid carcinoma: benefits and limits of radioiodine therapy. J Clin Endocrinol Metab 2006;91(8):2892-9.

6. Mazzaferri EL, Jhiang SM. Long-term impact of initial surgical and medical therapy on papillary and follicular thyroid cancer. Am J Med 1994;97(5):418-28.

7. Mandel SJ, Mandel L. Radioactive iodine and the salivary glands. Thyroid 2003;13(3):265-71.

8. Lamartina L, Cooper DS. Radioiodine remnant ablation in low-risk differentiated thyroid cancer: the "con" point of view. Endocrine 2015;50(1):67-71.

9. Ain KB. Radioiodine-remnant ablation in low-risk differentiated thyroid cancer: pros. Endocrine 2015;50(1):61-6.

10. Blumhardt R, Wolin EA, Phillips WT, Salman UA, Walker RC, Stack BC Jr, Metter D. Current controversies in the initial post-surgical radioactive iodine therapy for thyroid cancer: a narrative review. Endocr Relat Cancer 2014;21(6):R473-84.

11. Dohán O, De la Vieja A, Paroder V, Riedel C, Artani M, Reed M, et al. The Sodium/lodide Symporter (NIS): Characterization, Regulation, and Medical Significance. Endocr Rev 2003;24(1):48-77.

12. Mares J. Fyziologie trávení a vstřebávání. In: Trojan S, Langmeier M, editors. Lékařská fyziologie. Praha: Grada Publishing; 2003. p.323327.
13. Walter MA, Turtschi CP, Schindler C, Minnig P, Müller-Brand J, Müller $B$. The dental safety profile of high-dose radioiodine therapy for thyroid cancer: long-term results of a longitudinal cohort study. J Nucl Med 2007;48(10):1620-5.

14. Alexander C, Bader JB, Schaefer A, Finke C, Kirsch CM. Intermediate and long-term side effects of high-dose radioiodine therapy for thyroid carcinoma. J Nucl Med 1998;39(9):1551-4.

15. Grewal RK, Larson SM, Pentlow CE, Pentlow KS, Gonen M, Qualey R, Natbony L, Tuttle RM. Salivary gland side effects commonly develop several weeks after initial radioactive iodine ablation. J Nucl Med 2009;50(10):1605-10.

16. Hollingsworth B, Senter L, Zhang $X$, Brock GN, Jarjour W, Nagy R, Brock P, Coombes KR, Kloos RT, Ringel MD, Sipos J, Lattimer I, Carrau $\mathrm{R}$, Jhiang SM. Risk Factors of 1311-Induced Salivary Gland Damage in Thyroid Cancer Patients. J Clin Endocrinol Metab 2016;101(11):40853.

17. Jentzen W, Hobbs RF, Stahl A, Knust J, Sgouros G, Bockisch A. Pretherapeutic (124)I PET(/CT) dosimetry confirms low average absorbed doses per administered (131)I activity to the salivary glands in radioiodine therapy of differentiated thyroid cancer. Eur J Nucl Med Mol Imaging 2010;37(5):884-95.

18. Liu B, Huang R, Kuang A, Zhao Z, Zeng Y, Wang J, Tian R. lodine kinetics and dosimetry in the salivary glands during repeated courses of radioiodine therapy for thyroid cancer. Med Phys Oct;38(10):5412-9.

19. European Association of Nuclear Medicine. Internal Dosimetry Task Force Report on: Treatment planning For Molecular Radiotherapy Potential And Prospects. [cited 2019 Feb 18]. Available from: https:// www.eanm.org/publications/idtf-report/

20. Haugen BR, Alexander EK, Bible KC, Doherty GM, Mandel SJ, Nikiforov YE, Pacini F, Randolph W, Sawka AM, Schlumberger M, Schuff KG, Sherman SI, Sosa JA, Steward DL, Tuttle RM, Wartofsky L. 2015 American Thyroid Association Management Guidelines for Adult Patients with Thyroid Nodules and Differentiated Thyroid Cancer: The American Thyroid Association Guidelines Task Force on Thyroid Nodules and Differentiated Thyroid Cancer. Thyroid 2016;26(1):1-133.

21. Klutmann S, Bohuslavizki KH, Kröger S, Bleckmann C, Brenner W, Mester J, Clausen M. Quantitative salivary gland scintigraphy. J Nucl Med Technol 1999;27(1):20-6.

22. Bohuslavizki KH, Brenner W, Lassmann S, Tinnemeyer S, Tönshoff G, Sippel C, Wolf H, Clausen M, Henze E. Quantitative salivary gland scintigraphy in the diagnosis of parenchymal damage after treatment with radioiodine. Nucl Med Commun 1996;17(8):681-6.

23. Almeida JP, Sanabria AE, Lima EN, Kowalski LP. Late side effects of radioactive iodine on salivary gland function in patients with thyroid cancer. Head Neck 2011;33(5):686-90.

24. Solans R, Bosch JA, Galofré P, Porta F, Roselló J, Selva-O'Callagan A, Vilardell M. Salivary and lacrimal gland dysfunction (sicca syndrome) after radioiodine therapy. J Nucl Med 2001;42(5):738-43.

25. Wu JQ, Feng HJ, Ouyang W, Sun YG, Chen P, Wang J, Xian JL, Huang LH. Systematic evaluation of salivary gland damage following I-131 therapy in differentiated thyroid cancer patients by quantitative scintigraphy and clinical follow-up. Nucl Med Commun 2015;36(8):819-26.

26. Maruoka Y, Baba S, Isoda T, Kitamura Y, Abe K, Sasaki M, Honda H. A Functional Scoring System Based on Salivary Gland Scintigraphy for Evaluating Salivary Gland Dysfunction Secondary to (131)I therapy in Patients with Differentiated Thyroid Carcinoma. J Clin Diagn Res 2017;11(8):TC23-8.

27. Hermann GA, Vivino FB, Shnier D, Krumm RP, Mayrin V, Shore JB. Variability of quantitative scintigraphic salivary indices in normal subjects. J Nucl Med1998;39(7):1260-3. 